\title{
To: Gerstein HC, Pogue J, Mann JFE, Lonn E, Dagenais GR, McQueen M, Yusuf S, for the HOPE investigators (2005) The relationship between dysglycaemia and cardiovascular and renal risk in diabetic and non-diabetic participants in the HOPE study: a prospective epidemiological analysis. Diabetologia 48:1749-1755
}

Received: 31 October 2005 / Accepted: 31 October 2005 / Published online: 8 February 2006

(C) Springer-Verlag 2006

\begin{abstract}
Abbreviations FPG: fasting plasma glucose - UKPDS: UK Prospective Diabetes Study
\end{abstract}

To the Editor,

In their paper [1], Gerstein and colleagues employ the Heart Outcomes Prevention Evaluation (HOPE) study data in an attempt to pursue their contention that 'dysglycaemia' is a risk factor for cardiovascular disease that is independent of recognized categories of glucose intolerance [2]. Their data in diabetic patients, used to assess the relationships of both cardiovascular disease and nephropathy to mean updated glycated haemoglobin levels, are very similar to those from the UK Prospective Diabetes Study (UKPDS) [3], which evaluated a similar number of patients, with glycaemic levels being some 3-4 times more relevant for microvascular than for macrovascular endpoints. I would contend, however, that by attempting to prove a 'dysglycaemia' hypothesis (i.e. a continuous relationship between glycaemia and outcome) in non-diabetic subjects, they have ignored one of the conceptual tenets of scientific methodology [4].

The analyses of the 'progressive relationship' between baseline fasting plasma glucose (FPG) and relative hazards of four outcomes in 2,950 diabetic and non-diabetic subjects are shown in Fig. 2 of the article adjusted for age and sex, and in Table 3 with a series of additional adjustments [1]. These analyses were performed using Cox regression models with a logarithmic transformation of

\footnotetext{
J. S. Yudkin $(\bowtie)$

Department of Medicine,

Royal Free and University College London Medical School,

Holborn Union Building, Archway Campus,

2-10 Highgate Hill,

London N19 5LW, UK

e-mail: j.yudkin@ucl.ac.uk

Tel.: +44-207-2885051

Fax: +44-207-2883382
}

FPG as a continuous variable. Yet it is apparent from the figure that the relationships of baseline FPG with cardiovascular events (Fig. 2a), nephropathy (Fig. 2c), and death (Fig. 2d) are anything but linear: for each outcome, the relative hazard of those in the first three quintiles is very similar. So rather than showing a progressive risk, these findings are compatible with a threshold effect. Thus, for cardiovascular risk, the data show a threshold of risk starting in quintile 4 (FPG $6.2-8.5 \mathrm{mmol} / \mathrm{l})$, associated with a relative hazard of around 1.3; the relative hazard is increased to around 1.7 for subjects in quintile 5 (FPG $>8.6 \mathrm{mmol} / \mathrm{l}$ ). For nephropathy, these quintiles show relative hazards of around 2.5 and 8 , respectively. These observations might suggest that the threshold for risk is lower for macrovascular than for microvascular disease, something that has been recognised for some 30 years, and led, in 1980, to the introduction of a category of impaired glucose tolerance [5]. However, as quintile 4 includes some subjects with diabetes, as well as subjects with impaired fasting glucose, it is not possible to relate the data in these subjects to categories of glucose intolerance less severe than diabetes.

The use of a Cox regression model treats the data as if the risk is continuous and graded, so that the apparent $10 \%$ increase in (age- and sex-adjusted) risk of cardiovascular disease per $1 \mathrm{mmol} / \mathrm{l}$ increase in FPG is based on the excess risk in the two highest quintiles. The authors do not report any additional analyses, e.g. use of an additional quadratic term, to assess whether the data were more compatible with a threshold effect. They do mention, however, that self-reported diabetes (of a mean of 10 years' duration) somewhat reduces the relationship of baseline FPG with cardiovascular disease.

There is overwhelming evidence that diabetes is associated with an approximately two-fold excess risk of cardiovascular disease, and that this excess is not ex- 
plained by conventional risk factors. There is also substantial evidence that lesser degrees of glucose intolerance, particularly as measured after a glucose load [6-8], also predict excess cardiovascular disease. While we have long known that this excess is not explained by blood pressure, dyslipidaemia or obesity, what remains unclear is whether other, unmeasured, risk factors - such as low grade inflammation [9] or endothelial dysfunction [10] - might represent a common antecedent. The concept that the excess risk is the consequence of 'dysglycaemia' would require evidence of reversibility (what Bradford Hill referred to as experimentation [11]), and the UKPDS [12] represents an important negative test of the hypothesis.

\section{References}

1. Gerstein HC, Pogue J, Mann JFE et al (2005) The relationship between dysglycaemia and cardiovascular and renal risk in diabetic and non-diabetic participants in the HOPE study: a prospective epidemiological analysis. Diabetologia 48:17491755

2. Gerstein HC, Yusuf S (1996) Dysglycaemia and risk of cardiovascular disease. Lancet 347:949-950

3. Stratton IM, Adler AI, Neil HAW et al (2000) Association of glycaemia with macrovascular and microvascular complications of type 2 diabetes (UKPDS 35): prospective observational study. BMJ 321:405-412
4. Popper KR (1963) Conjectures and Refutations. Routledge and Kegan Paul, London

5. World Health Organization Expert Committee on Diabetes Mellitus. Second Report. WHO, Geneva, 1980

6. DECODE study group on behalf of the European Diabetes Epidemiology Study Group (1999) Glucose tolerance and mortality: comparison of WHO and American Diabetes Association diagnostic criteria. Lancet 354:614-621

7. de Vegt F, Dekker JM, Ruhe HG et al (1999) Hyperglycaemia is associated with all-cause and cardiovascular mortality in the Hoorn population: the Hoorn Study. Diabetologia 42:926-931

8. Balkau B, Shipley M, Jarrett RJ et al (1998) High blood glucose concentration is a risk factor for mortality in middleaged nondiabetic men. 20 year follow up in the Whitehall Study, the Paris Prospective Study, and the Helsinki Policemen Study. Diabetes Care 21:360-367

9. Yudkin JS, Stehouwer CDA, Emeis JJ, Coppack SW (1999) C-reactive protein in healthy subjects: association with obesity, insulin resistance, and endothelial dysfunction. Arterioscler Thromb Vasc Biol 19:972-978

10. Pinkney J, Coppack SW, Yudkin JS (1997). Endothelial dysfunction: cause of the insulin resistance syndrome? Diabetes 46 (Suppl 2):S9-S13

11. Bradford Hill A (1965) The environment and disease: association or causation. Proc R Soc Med 58:295-300

12. UK Prospective Diabetes Study (UKPDS) Group (1998) Intensive blood-glucose control with sulphonylureas or insulin compared with conventional treatment and risk of complications in patients with type 2 diabetes (UKPDS 33). Lancet 352:837-853 